\title{
As Cores do Tabagismo: Relação entre Raça e Consumo de Tabaco no Brasil
}

doi: https://doi.org/10.32635/2176-9745.RBC.2022v68n1.1552

\author{
The Colors of Smoking: Relationship Between Race and Tobacco Use in Brazil \\ Los Colores del Fumar: Relación entre Raza y Uso de Tabaco en Brasil
}

André Luiz Oliveira da Silva '; Caroline de Lima Mota²; Renata Aparecida Pereira ${ }^{3}$; Simone Mitri Nogueira ${ }^{4}$; Josino Costa Moreira ${ }^{5}$

\section{INTRODUÇÃO}

A relação entre raça (e etnicidade) e adoecimento no Brasil é bastante sólida e evidente. A cor da pele de um indivíduo influencia significativamente na sua vida, no seu adoecimento e morte ${ }^{1}$. Como exemplo atual sobre o tema, cabe mencionar a relação entre a mortalidade da doença pelo coronavírus 2019 (do inglês, coronavirus disease 2019 - covid-19) e a raça, cuja população de pretos tem uma taxa de mortalidade mais alta do que os brancos ${ }^{2}$.

No caso do tabagismo, uma série de fatores sociais, ambientais, psicológicos e genéticos estão associados à dependência da nicotina e às doenças tabaco-relacionadas. Idade, gênero, classe social, nível educacional, localização geográfica e raça/etnicidade são alguns dos fatores que podem influenciar o comportamento tabágico ${ }^{3}$. Entre esses fatores, a relação entre raça/etnicidade e tabagismo talvez seja uma das mais inexploradas e menos discutidas no âmbito do controle do tabaco no Brasil.

A escassez de pesquisas no Brasil que explorem o impacto de raça e etnia no consumo do tabaco e as suas consequências faz com que esse assunto seja ainda mais relevante, principalmente considerando que, embora o consumo de tabaco tenha diminuído ao longo dos anos, o hábito tabágico ainda tem maior prevalência entre pretos e partos do que em brancos ${ }^{4}$.

A própria questáo do racismo estrutural como determinante social ainda é subestimada e, em alguns casos, não é considerada. Estudos analisando o seu impacto no tabagismo são escassos.

\section{QUE SE SABE SOBRE 0 TEMA?}

Para subsidiar essa discussão e ilustrar como essa temática é pouco estudada no Brasil, realizou-se uma análise qualitativa retrospectiva em junho de 2021, usando as bases de dados PubMed (MEDLINE) e SciELO para as buscas bibliográficas. Para tal, utilizaram-se os seguintes termos de pesquisa: (Smoking) AND (Race) AND (Brazil) OR (Smoking) AND (Afro-American) AND (Brazil) OR (Smoking AND "Special Populations" AND Brazil). Todo o período disponível nas bases de dados até o dia da pesquisa foi considerado. Os critérios de inclusão foram artigos em língua inglesa, portuguesa e espanhola, publicados pelas respectivas bases de dados que tratavam sobre o assunto. Artigos duplicados, não disponíveis na íntegra e em outras línguas, foram excluídos. A pesquisa referente aos termos mencionados resultou em três artigos que discutiam a relação etnicidade/raça e tabagismo no Brasil.

Adicionalmente, realizou-se uma busca bibliográfica, a fim de discutir a contribuição genética no tema abordado no presente trabalho. Foram utilizados os termos de pesquisa citados anteriormente, adicionando os descritores: (genetic OR polymorphism). Seguindo os mesmos critérios de inclusão e exclusão já definidos, foram selecionados dois artigos classificados dentro do escopo, de acordo com os critérios estabelecidos.

Além disso, também foram incluídos artigos considerados relevantes para o fornecimento do embasamento técnico-científico, segundo o conteúdo de caráter inédito ou na forma de revisões sobre o tema.

\section{DESENVOLVIMENTO}

Internacionalmente, especialmente nos Estados Unidos da América (EUA), estudos apontam que a origem étnica representa um fator de risco para o tabagismo, onde, por exemplo, a prevalência do tabagismo em hispânicos é de $10,1 \%$, enquanto, em nativos americanos, é duas vezes maior, $21,9 \%{ }^{5}$.

No Brasil, pesquisas apontam que a prevalência do tabagismo é maior entre pretos e pardos ${ }^{4}$. Segundo a Pesquisa Nacional de Saúde de $2019^{4}$, o predomínio do

Agência Nacional de Vigilância Sanitária (Anvisa). Fundação Oswaldo Cruz, Escola Nacional de Saúde Pública Sérgio Arouca, Centro de Estudos da Saúde do Trabalhador e Ecologia Humana (Fiocruz/Ensp/Cesteh). Rio de Janeiro (RJ), Brasil. E-mail: andre.sp.ensp@gmail.com. Orcid iD: https://orcid.org/0000-0003-4768-959X

2,4,5Fiocruz/Ensp/Cesteh. Rio de Janeiro (RJ), Brasil. E-mails: carolinelima_bio@yahoo.com.br; simitri@ensp.fiocruz.br; josino.moreira@fiocruz.br. Orcid iD: https:// orcid.org/0000-0002-2021-6525; Orcid iD: https://orcid.org/0000-0003-2400-333X; Orcid iD: https://orcid.org/0000-0002-7457-2920

${ }^{3}$ Fiocruz. Instituto Nacional de Controle de Qualidade em Saúde (INCQS). Rio de Janeiro (RJ), Brasil. E-mail: renattaali@gmail.com. Orcid iD: https://orcid.org/00000001-9146-2996

Endereço para correspondência: André Luiz Oliveira da Silva. Laboratório de Toxicologia do Cesteh/Ensp/Fiocruz. Rua Leopoldo Bulhões, 1480 - Manguinhos. Rio de Janeiro (RJ), Brasil. CEP 21041-210. E-mail: andre.sp.ensp@gmail.com 
tabagismo em pretos era de 13,7\%, 13,5\% em pardos e $11,8 \%$ em brancos. Além disso, pretos e pardos também possuíam menor nível de escolaridade e de renda, conforme verificado na Tabela 1 . Esses dados representam um dos vários indicadores que revelam a desigualdade racial ainda presente na sociedade brasileira.

Baixa escolaridade, menor renda e menor urbanização são fatores de risco para o tabagismo no Brasil ${ }^{4-8}$, o que pode ser atribuído, em parte, a fatores sociais, considerando que pretos e pardos constituem a maior parcela da população com menor renda e nível educacional no país, contribuindo para a vulnerabilidade dessa população?.

Embora não tenha sido publicado nenhum estudo no Brasil, que avaliasse os impactos à saúde, a cessação ou o padrão de uso do tabaco relacionado à raça/etnicidade, algumas evidências apontam impactos do tabagismo na saúde da população com ancestralidade africana no Brasil, como, por exemplo, a maior mortalidade por doença cerebrovascular em pretos ${ }^{10}$.

Esses dados apresentam semelhança com os dados encontrados nos EUA, onde os afrodescendentes, apesar de começarem a fumar mais tarde e consumirem uma quantidade menor de cigarros que os brancos, estão mais propensos a morrer por doenças tabaco-relacionadas, entre elas, os acidentes vasculares cerebrais ${ }^{11,12}$.

No Brasil, inexistem estudos sobre o desejo de parar de fumar entre pretos e a exposição dessas populaçóes ao fumo passivo. A literatura aponta que, nos EUA, os afro-americanos são os que mais expressam desejo de parar de fumar, contudo, são os que menos têm sucesso, quando comparados com hispânicos, brancos e asiáticos ${ }^{13}$. Adicionalmente, os não fumantes desse grupo seriam os mais sujeitos à exposição ao fumo passivo ${ }^{14}$.

Nos EUA, mais de $70 \%$ dos fumantes afro-americanos mostraram preferir os cigarros mentolados aos convencionais, além da alta prevalência de jovens pretos consumindo esse tipo de cigarro, quando comparados com outras etnias ${ }^{15}$. No Brasil, não existem estudos dessa natureza.

Os motivos dessa preferência pelos cigarros mentolados nos EUA são frequentemente associados às estratégias utilizadas pela Indústria do Tabaco (IT) direcionadas a tais grupos. Essas estratégias remontam à década de 1960, período que coincide com a urbanizaçáo da população afrodescendente nos $\mathrm{EUA}^{15}$ e poderia explicar as diferenças nos padróes de uso de tabaco ${ }^{16}$.

A literatura sugere ainda que os afrodescendentes que utilizam cigarros mentolados têm menor taxa de sucesso na cessação do tabagismo ${ }^{17}$. As razóes ou os mecanismos envolvidos nesse processo ainda náo claros.

No Brasil, até o momento, não foram identificadas estratégias da IT específicas para populaçóes de pretos, apesar de a IT ainda promover a divulgaçáo de seus produtos de maneira insidiosa, especialmente entre os mais jovens, como, por exemplo, em festivais de música e redes sociais, conforme denunciado por uma matéria da Folha de São Paulo ${ }^{18}$, da qual pode-se inferir que essas estratégias poderiam ser facilmente direcionadas a grupos específicos.

Um outro fator menos estudado, mas que também poderia contribuir para esse cenário, é a genética; alguns estudos apontam que este seria um importante componente para o comportamento do tabagismo e mostram como as variaçôes genéticas interindividuais poderiam interferir na resposta aos fatores de risco ambientais ${ }^{19,20}$.

Um estudo que estimou a influência genética no tabagismo na população brasileira observou que a hereditariedade contribuiu em mais de $50 \%$ para a iniciação, e variou de $23 \%$ a $32 \%$ para persistência e quantidade de cigarros consumidos por $\mathrm{dia}^{21}$.

Em adição a isso, a etnicidade tem sido apontada como um possível fator de influência na relação entre a variabilidade genética e o tabagismo ${ }^{22-24}$.

Tabela 1. Estrutura, prevalência do tabagismo, escolaridade e renda dos grupos raciais no Brasil

\begin{tabular}{|c|c|c|c|c|c|c|}
\hline & $\begin{array}{c}\text { Percentual } \\
\text { da } \\
\text { população } \\
\text { brasileira }\end{array}$ & $\begin{array}{c}\text { Prevalência } \\
\text { do } \\
\text { tabagismo } \\
(\%)\end{array}$ & $\begin{array}{c}\text { Analfabetismo } \\
(\%)\end{array}$ & $\begin{array}{l}\text { Pessoas com } 25 \\
\text { anos ou mais } \\
\text { com o Ensino } \\
\text { Médio completo } \\
(\%)\end{array}$ & $\begin{array}{l}\text { Rendimento } \\
\text { médio } \\
\text { mensal (RS) }\end{array}$ & $\begin{array}{l}\text { Percentual } \\
\text { de pessoas } \\
\text { abaixo da } \\
\text { linha da } \\
\text { pobreza } \\
\text { (US\$ 1,90/dia) }\end{array}$ \\
\hline Brancos & 47,43 & 11,8 & 3,9 & 55,8 & 2.796 & 3,6 \\
\hline Pretos & 7,61 & 13,7 & \multirow{2}{*}{$9,1^{*}$} & \multirow{2}{*}{$40,3^{*}$} & \multirow{2}{*}{$1.608^{*}$} & \multirow{2}{*}{$8,8^{*}$} \\
\hline Pardos & 43,13 & 13,5 & & & & \\
\hline Amarelo & 1,09 & Sem dados & Sem dados & Sem dados & Sem dados & Sem dados \\
\hline Índios & 0,43 & Sem dados & Sem dados & Sem dados & Sem dados & Sem dados \\
\hline
\end{tabular}

$\left(^{*}\right)$ Dados de pretos e pardos apresentados pelo IBGE de forma conjunta.

Fonte: Instituto Brasileiro de Geografia e Estatística ${ }^{4}$ e Malta et al. 
$\mathrm{Na}$ população brasileira, que tem um perfil étnico heterogêneo e altamente miscigenado ${ }^{25}$, a influência da etnia na relação genética-tabagismo é complexa e ainda pouco estudada ${ }^{26}$. Os estudos predominantemente avaliam a variável raça como uma característica autorreferida, sem o uso adicional de marcadores genéticos relacionados à ancestralidade, marcadores estes que contribuiriam para minimizar as limitaçóes desse tipo de estudo no contexto da miscigenação da população brasileira ${ }^{1,26}$. Apesar da influência da genética, ainda assim, as disparidades socioeconômicas podem representar um fator de maior peso do que a ancestralidade em relação ao tabagismo e suas consequências ${ }^{26}$.

Os dados relativos aos afrodescendentes brasileiros e ao tabagismo são escassos e tornam esse grupo populacional invisível para a elaboração de políticas públicas. Adicionalmente a esses achados, foi publicada uma reportagem denunciando uma suposta censura, por parte do Ministério da Saúde, de dados sobre a saúde da população afrodescendente brasileira ${ }^{27}$. Assim sendo, a falta de estudos sobre o tema não reflete a dimensão do problema.

\section{CONCLUSÃO}

Dessa forma, o tabagismo e seus impactos em populaçóes de origem africana têm influência dos determinantes sociais, do racismo estrutural, e dos fatores genéticos. Mais pesquisas seriam necessárias para apontar o peso de cada fator (escolaridade, renda e urbanização, por exemplo) que, nessas populaçóes, sabidamente são desfavoráveis ao quadro de dependência ao tabaco. Por outro lado, é inequívoco que negros fumam mais do que outros grupos raciais.

A IT tem várias campanhas de publicidade com público-alvo diferenciado. A falta de uma análise sobre campanhas direcionadas a negros não significa que estas não existam. De fato, seria um tipo de pesquisa também. A dificuldade em diferenciar raça/etnia de nível de escolaridade e de rendimento é uma outra questão que merece mais atenção.

A delimitação desses fatores é importante, pois contribui para o estabelecimento de novas estratégias para o enfretamento do tabagismo, uma vez que o modelo vigente é baseado em padrões de comunicações únicos e não considera os diferentes aspectos sociais, culturais e raciais da população que, apesar de ser considerado de sucesso em nível global, pode ser aprimorado.

Ter ancestralidade africana no Brasil, seja pela associação com fatores de risco como menor renda e escolaridade, aliada a uma possível contribuição genética, é uma espécie de tempestade perfeita para o tabagismo e para as doenças tabaco-relacionadas, entretanto, em virtude dos poucos estudos sobre o tema, essa populaçáo passa desapercebida pelos formuladores de políticas de saúde em relação ao tabaco e por pesquisadores do tema. Portanto, pesquisas referentes a esse grupo populacional, a fim de minimizar os impactos causados pelo tabaco nessas populaçóes e, dessa forma, aprimorar ainda mais a já exitosa Política de Controle do Tabaco no Brasil, são de extrema importância.

\section{CONTRIBUIÇÕES}

André Luiz Oliveira da Silva contribuiu substancialmente na concepção e/ou no planejamento do estudo; na obtenção, análise e/ou interpretação dos dados; na redação e/ou revisão crítica. Caroline de Lima Mota, Renata Aparecida Pereira, Simone Mitri Nogueira e Josino Costa Moreira contribuíram substancialmente na obtenção, análise e/ou interpretação dos dados; na redação e/ou revisão crítica. Todos os autores aprovaram a versão final a ser publicada.

\section{AGRADECIMENTOS}

À Agência Nacional de Vigilância Sanitária (Anvisa), à Fundação Oswaldo Cruz (Fiocruz), ao Instituto Nacional de Câncer José Alencar Gomes da Silva (INCA), à Coordenação de Aperfeiçoamento de Pessoal de Nível Superior (Capes) e ao Conselho Nacional de Desenvolvimento Científico e Tecnológico (CNPq). Este texto reflete única e exclusivamente a opiniāo e os pensamentos dos autores, com base nas evidências científicas disponíveis no momento, e não representa qualquer diretriz e/ou opiniáo institucional da Anvisa, da Fiocruz, do INCA, do Ministério da Saúde ou do Governo Brasileiro.

\section{DECLARAÇÃO DE CONFLITO DE INTERESSES}

Nada a declarar.

\section{FONTES DE FINANCIAMENTO}

Coordenação de Aperfeiçoamento de Pessoal de Nível Superior - Brasil (Capes) - Código de Financiamento 001.

\section{REFERÊNCIAS}

1. Chor D. Desigualdades em saúde no Brasil: é preciso ter raça. Cad Saúde Pública. 2013;29(7):1272-5. doi: https://doi.org/10.1590/S0102-311X2013000700002

2. Instituto Pólis [Internet]. São Paulo: Instituto Pólis; c2020. Raça e Covid no município de São Paulo; 2020 jul. [acesso 2020 out 24]. Disponível em: https://polis. org.br/estudos/raca-e-covid-no-msp/ 
3. Mentis AA. Social determinants of tobacco use: towards an equity lens approach. Tob Prev Cessat. 2017;3:7. doi: https://doi.org/10.18332/tpc/68836

4. Instituto Brasileiro de Geografia e Estatística. Pesquisa nacional de saúde: 2019: percepção do estado de saúde, estilos de vida, doenças crônicas e saúde bucal: Brasil e grandes regiōes [Internet]. Rio de Janeiro: IBGE; 2020 [acesso $2021 \mathrm{fev}$ 10]. Disponível em: https://biblioteca. ibge.gov.br/index.php/biblioteca-catalogo?view=detalh es\&id=2101764

5. Centers for Disease Control and Prevention [Internet]. Atlanta (GA): CDC. American Indians/Alaska natives and tobacco use; [revised 2021 Dec 3; cited 2021 May 2]. Available from: https://www.cdc.gov/tobacco/disparities/ american-indians/index.htm

6. Bazotti A, Finokiet M, Conti IL, et al. Tabagismo e pobreza no Brasil: uma análise do perfil da populaçáo tabagista a partir da POF 2008-2009. Ciênc Saúde Colet. 2016;21(1):45-52. doi: https://doi.org/10.1590/141381232015211.16802014

7. Malta DC, Vieira ML, Szwarcwald CL, et al. Tendência de fumantes na população Brasileira segundo a Pesquisa Nacional de Amostra de Domicílios 2008 e a Pesquisa Nacional de Saúde 2013. Rev Bras Epidemiol. 2015;18(Suppl 2):45-56. doi: https://doi. org/10.1590/1980-5497201500060005

8. Muzi CD, Figueiredo VC, Luiz RR. Gradiente urbanorural no padrão de consumo e cessaçáo do tabagismo no Brasil. Cad Saúde Pública. 2018;34(6):e00077617. doi: https://doi.org/10.1590/0102-311X00077617

9. Instituto Brasileiro de Geografia e Estatística. Desigualdades sociais por cor ou raça no Brasil. Estudos e Pesquisas. Informação Demográfica e Socioeconômica [Internet]. 2019 [acesso 2020 out 29];41:1-12. Disponível em: https://biblioteca.ibge.gov. br/visualizacao/livros/liv101681_informativo.pdf

10. Lotufo PA, Bensenor IJM. Raça e mortalidade cerebrovascular no Brasil. Rev Saúde Pública. 2013;47(6):1201-4. doi: https://doi.org/10.1590/ S0034-8910.2013047004890

11. Department of Health and Human Services (US). Tobacco use among U.S. racial/ethnic minority groups: African Americans, American Indians and Alaska Natives, Asian Americans and Pacific Islanders, and Hispanics: a report of the surgeon general [Internet]. Atlanta (GA): Department of Health and Human Services, Centers for Disease Control and Prevention, National Center for Chronic Disease Prevention and Health Promotion, Office on Smoking and Health; 1998 [cited 2020 Oct 24]. Available from: https://www. cdc.gov/tobacco/data_statistics/sgr/1998/complete_ report/index.htm

12. Kochanek KD, Murphy SL, Xu J, et al. Deaths: final data for 2014. Natl Vital Stat Rep. 2016;65(4):1-122.
13. Babb S, Malarcher A, Schauer G, et al. Quitting smoking among adults - United States, 2000-2015. MMWR Morb Mortal Wkly Rep. 2017;65(52):1457-64. doi: https://doi.org/10.15585/mmwr.mm6552a1

14. Tsai J, Homa DM, Gentzke AS, et al. Exposure to secondhand smoke among nonsmokers - United States, 1988-2014. MMWR Morb Mortal Wkly Rep. 2018;67(48):1342-6. doi: https://doi.org/10.15585/ mmwr.mm6748a3

15. Gardiner PS. The African Americanization of menthol cigarette use in the United States. Nicotine Tob Res. 2004;6(Suppl 1):S55-65. doi: https://doi.org/10.1080 /14622200310001649478

16. Yerger VB, Przewoznik J, Malone RE. Racialized geography, corporate activity, and health disparities: tobacco industry targeting of inner cities. J Health Care Poor Underserved. 2007;18(4 Suppl):10-38. doi: https:// doi.org/10.1353/hpu.2007.0120

17. Smith PH, Assefa B, Kainth S, et al. Use of mentholated cigarettes and likelihood of smoking cessation in the United States: a meta-analysis. Nicotine Tob Res. 2020;22(3):307-16. doi: https://doi.org/10.1093/ntr/ ntz067

18. Watanabe P. Festivais de música no Brasil são palco para promover cigarro entre jovens. Folha de São Paulo [Internet]. 2018 ago 30 [acesso 2018 nov 7]. Disponível em: https://www1.folha.uol.com.br/ equilibrioesaude/2018/08/festivais-de-musica-no-brasilsao-palco-para-promover-cigarro-entre-jovens.shtml

19. Ducci F, Goldman D. The genetic basis of addictive disorders. Psychiatr Clin North Am. 2012;35(2):495519. doi: https://doi.org/10.1016/j.psc.2012.03.010

20. Munafò M, Clark T, Johnstone E, et al. The genetic basis for smoking behavior: a systematic review and meta-analysis. Nicotine Tob Res. 2004;6(4):583-97. doi: https://doi.org/10.1080/14622200410001734030

21. Horimoto AR, Oliveira CM, Giolo SR, et al. Genetic analyses of smoking initiation, persistence, quantity, and age-at-onset of regular cigarette use in Brazilian families: the Baependi Heart Study. BMC Med Genet. 2012;13(1):9. doi: https://doi.org/10.1186/1471-2350-13-9

22. Ohmoto M, Takahashi T, Kubota Y, et al. Genetic influence of dopamine receptor, dopamine transporter, and nicotine metabolism on smoking cessation and nicotine dependence in a Japanese population. BMC Genet. 2014;15(1):151. doi: https://doi.org/10.1186/ s12863-014-0151-2

23. Vasconcelos GM, Struchiner CJ, Suarez-Kurtz G. CYP2A6 genetic polymorphisms and correlation with smoking status in Brazilians. Pharmacogenomics J. 2005;5(1):42-8. doi: https://doi.org/10.1038/ sj.tpj. 6500290

24. Pérez-Rubio G, Córdoba-Lanús E, Cupertino $P$, et al. Role of genetic susceptibility in nicotine addiction and 
chronic obstructive pulmonary disease. Rev Investig Clín. 2019;71(1):36-54. doi: https://doi.org/10.24875/ RIC. 18002617

25. Lins TC, Vieira RG, Abreu BS, et al. Genetic composition of Brazilian population samples based on a set of twentyeight ancestry informative SNPs. Am J Hum Biol. 2010;22(2):187-92. doi: https://doi.org/10.1002/ ajhb.20976

26. Souza EST, Araújo LF, Alencar DO, et al. Does ethnic ancestry play a role in smoking? An Acad Bras Ciênc. 2015;87(1):447-53. doi: https://doi.org/10.1590/00013765201520140187

27. Valente R. Ministério da Saúde retira do ar estudo sobre saúde da população negra. UOL Notícias [Internet]. 2020 jun 10 [acesso 2020 out 26]. Disponível em: https://noticias.uol.com.br/colunas/rubensvalente/2020/06/10/censura-saude-populacao-negra. htm 